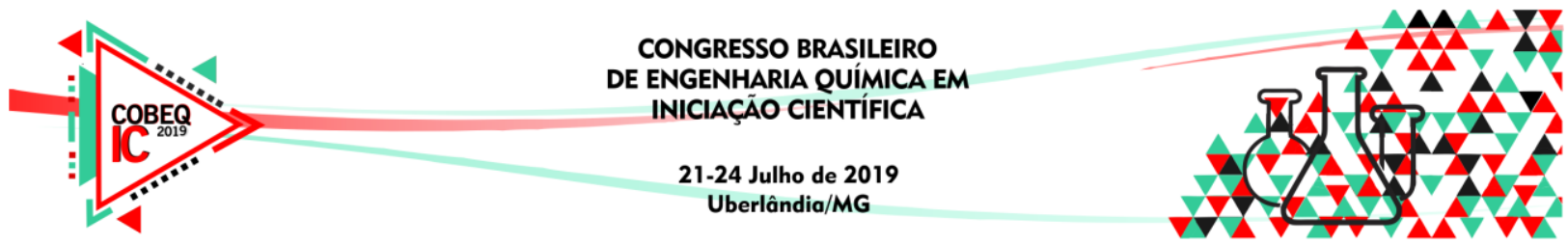

\title{
AVALIAÇÃO DA PRODUÇÃO DE FICOCIANINA POR Anabaena variabilis UTILIZANDO DIFERENTES FONTES DE CARBONO ORGÂNICO
}

\author{
W. R. CUNHA ${ }^{1}$, T. A. TEIXEIRA ${ }^{2}$, A. G. COTTAS ${ }^{2}$ e J. S. FERREIRA ${ }^{2}$ \\ ${ }^{1}$ Universidade Federal de Uberlândia, Instituto de Biotecnologia \\ ${ }^{2}$ Universidade Federal de Uberlândia, Faculdade de Engenharia Química \\ E-mail para contato: warlleyrcunha@gmail.com
}

\begin{abstract}
RESUMO - A ficocianina (FC) é uma das ficobiliproteínas, pigmentos encontrados em cianobactérias, que podem ser empregadas como corantes naturais, antioxidantes e marcadores fluorescentes. Este estudo teve como objetivo avaliar o efeito da suplementação do meio de cultivo basal (BG110) da cianobactéria Anabaena variabilis suplementando $1 \mathrm{~g} / \mathrm{L}$ da fonte de carbono orgânico, sendo testadas: glicose, lactose, frutose, sacarose e galactose, visando aumentar a produção de biomassa e de FC. O meio controle, utilizado no cultivo da cianobactéria produziu 1,61 $\mu \mathrm{g} \mathrm{FC} \mathrm{mL}^{-1}$ em 8 dias, enquanto os meios contendo glicose e galactose apresentaram um aumento de cerca de 6 vezes com concentrações de 10,26 $\mu \mathrm{g} \mathrm{FC} \mathrm{mL}^{-1}$ e $9,36 \mu \mathrm{g} \mathrm{FC} \mathrm{mL}^{-1}$, respectivamente. A produção de FC sofreu uma influencia menor ao seu suplementar o meio com lactose, com aumento de 3 vezes em relação ao meio basal $\left(5,31 \mu \mathrm{g} \mathrm{FC} \mathrm{mL} \mathrm{Fs}^{-1}\right)$. Os meios contendo frutose e sacarose não teve efeito sobre a produção de FC. Todos os meios suplementados com os açúcares apresentaram uma produção maior de biomassa com relação ao meio basal, com destaque para o meio com glicose.
\end{abstract}

\section{INTRODUÇÃO}

As cianobactérias são organismos fotossintéticos e produtores de ampla variedade de metabólitos secundários com aplicação biotecnológica. Dentre estes metabólitos, destacam-se as ficobiliproteínas que representam $50 \%$ do total de proteína celular das cianobactérias e podem ser aplicadas como antioxidantes, anticâncer e anti-inflamatórios. Quantitativamente, a ficocianina (FC) é a ficobiliproteína mais abundante nas cianobactérias. A síntese deste composto bioativo pode ser avaliada com o intuito de aumentar sua produção em escala industrial, ao abordar parâmetros como luz, composição do meio, pH, temperatura e fotoperíodo (PAGELS et al, 2019, MANIRAFASHA et al.,2016).

Mais especificamente, com relação à composição, deve-se ressaltar que a fonte de carbono influencia na formação principalmente dos macronutrientes, como carboidratos, lipídeos e proteínas (MANIRAFASHA et al.,2016). Atualmente, o meio mais empregado para cultivo de biomassa e de ficobiliproteínas é o meio mineral BG11 0 , constituindo um cultivo fotoautotrófico. No entanto, alguns pesquisadores têm estudado a influência de cultivos mixotróficos, em que a fonte de carbono é formada por substâncias minerais e orgânicas, de 


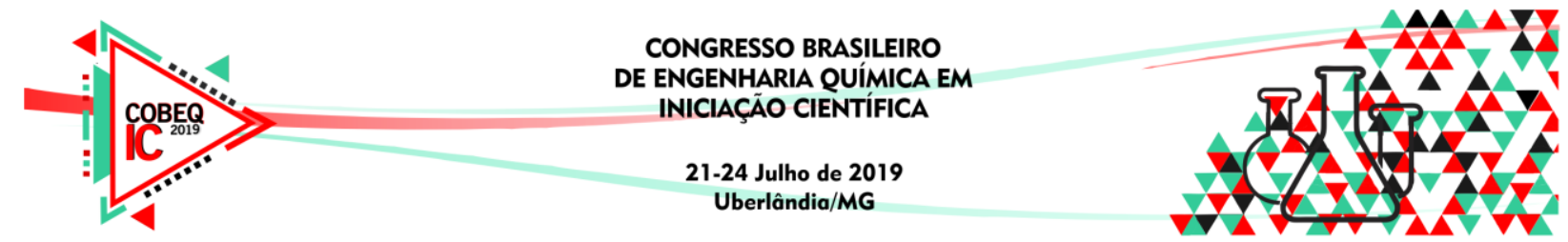

modo a aumentar a produtividade de biomassa e produtos, como por exemplo as ficobiliproteínas (FBP), de forma mais eficiente (BORSARI et al., 2007; RIZZO et al., 2015).

O presente trabalho teve por objetivo avaliar o efeito da suplementação do meio de cultivo basal da cianobactéria Anabaena variabilis (BG110) por fontes de carbono orgânico, utilizando glicose, lactose, frutose, sacarose e galactose, visando aumentar a produção de biomassa e de ficocianina (FC).

\section{MATERIAIS E MÉTODOS}

A cepa de Anabaena variabilis ATCC 29413, cedida pelo Laboratório de Cianobactérias e Ficotoxinas da FURG (Rio Grande, RS), foi cultivada em meio basal BG11 0 (JACINAVICIUS et al., 2013), em Erlenmeyers de 250 e $500 \mathrm{~mL}$, a $30^{\circ} \mathrm{C}$, luminosidade de 1.553 lux, com fotoperíodo de $12 \mathrm{~h}$, em ambiente aeróbico e com repiques periódicos de 14 dias.

Essa cepa mantida em meio basal (BG110) foi utilizada como inóculo para os ensaios de avaliação do efeito de diferentes fontes de carbono orgânico para sobre a produção de biomassa e ficocianina por fermentação. Os ensaios foram realizados em Erlenmeyers de 125 $\mathrm{mL}$, com volume útil de $50 \mathrm{~mL}$, com volume de inóculo de $20 \%$ (v/v) o que correspondeu a uma concentração celular inicial de 0,18 g/L. Foram preparados o meio basal sem adição de da fonte de carbono (controle) e com suplementação de $1 \mathrm{~g} / \mathrm{L}$ de açúcar. Os açúcares testados foram: glicose, lactose, frutose, sacarose e galactose. Cada condição foi realizada em duplicata.

Foram avaliadas concentração celular em 2, 4, 6 e 8 dias de fermentação e síntese de ficocianina ao final de 8 dias do processo. A determinação da biomassa foi realizada por espectrofotometria (Shimadzu UV mini-1240) no comprimento de onda de $610 \mathrm{~nm}$.

A quantificação dos açúcares foi realizada por cromatografia líquida de alto desempenho HPLC (High Performance Liquid Chromatography). A amostra foi filtrada em filtro $0,22 \mu \mathrm{m}$ e injetada no cromatógrafo marca Shimadzu modelo LC-20A Pronience, equipado com coluna SUPELCOGEL C-610H e detector de índice de refração, com solução de ácido fosfórico $(0,1 \%)$ usada como fase móvel, sendo a vazão da bomba de $0,5 \mathrm{~mL} / \mathrm{min}$, temperatura do forno de $32{ }^{\circ} \mathrm{C}$ e volume de injeção de $20 \mu \mathrm{L}$.

A extração de FC foi realizada por ciclos de congelamento e descongelamento combinados com ultrassom, sendo 5 ciclos de congelamento a $-15^{\circ} \mathrm{C}$ por $1,5 \mathrm{~h}$ e descongelamento no banho ultrassônico a $25^{\circ} \mathrm{C}$ por $30 \mathrm{~min}$ (COTTAS, 2019). A concentração de cada FC $\left(\mu \mathrm{g} \mathrm{mL}^{-1}\right)$ foi calculada de acordo com a Equação 1 (LIU et al., 2012):

$$
[F C]=\frac{A_{615}-0,474\left[A_{652}\right]}{5,34}
$$




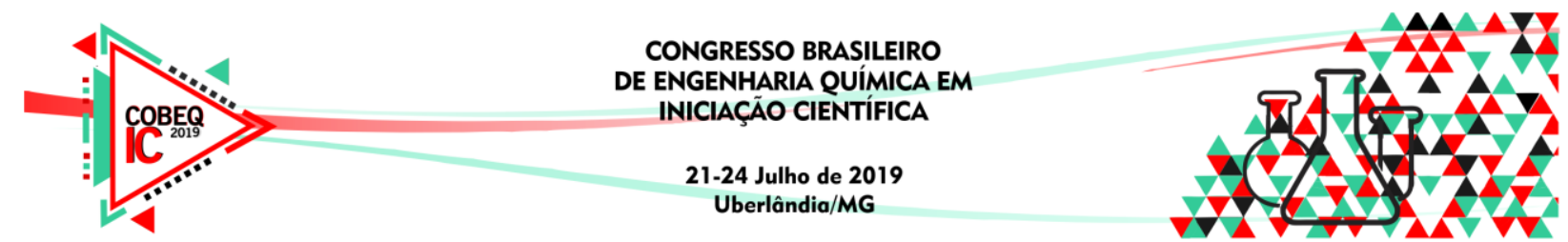

\section{RESULTADOS E DISCUSSÃO}

Observa-se pela Figura 1, o consumo das fontes de carbono nos diferentes meios de cultivo ao decorrer de 6 dias. Houve um consumo, nesta ordem crescente de 54\% para lactose, $57 \%$ para glicose, $62 \%$ para sacarose, $67 \%$ para galactose e $77 \%$ para frutose, indicando a cianobactéria de Anabaena variabilis pode assimilar todas as fontes de carbono testadas.

Figura 1 - Consumo de fonte de carbono durante 6 dias no cultivo da Anabaena variabilis. Glicose $1 \mathrm{~g} / \mathrm{L}(*)$, Lactose $1 \mathrm{~g} / \mathrm{L}(\boldsymbol{\Delta})$, Frutose $1 \mathrm{~g} / \mathrm{L}(\diamond)$, Sacarose $1 \mathrm{~g} / \mathrm{L}(\bullet)$, Galactose $1 \mathrm{~g} / \mathrm{L}(\square)$.



A variação da concentração de biomassa é apresentada na Figura 2. Verifica-se que, em todas condições de suplementação, houve aumento da concentração celular acima do o observado para o meio controle, sendo a glicose com maior valor obtido $(0,47 \mathrm{~g} / \mathrm{L})$, sendo 2 vezes maior que o meio controle. Deve-se ressaltar que a glicose não foi o açúcar mais consumido (Figura 1), mas sua conversão resultou em maios crescimento de biomassa.

Os dados do efeito da suplementação de açúcar ao meio basal (BG110) sobre a produção de ficocianina são mostrados na Figura 3.

Verifica-se que o cultivo da Anabaena variabilis, ao final de 8 dias, nos meios suplementados com glicose, lactose e galactose favoreceram a produção de FC atingindo teores acima do daquele observado para a condição controle. $O$ teor de ficocianina os meios glicose e galactose não apresentaram diferença significativa, tendo-se a concentração de 10,26 $\pm 1,74 \mu \mathrm{g} \mathrm{FC} \mathrm{mL}{ }^{-1}$ e 9,36 $\pm 0,19 \mu \mathrm{g} \mathrm{FC} \mathrm{mL}{ }^{-1}$, respectivamente, cerca de 6 vezes maior quando comparado ao meio controle. Para o meio contendo lactose, este aumento foi de 3 vezes, apresentando uma concentração de 5,31 $\pm 2,00 \mu \mathrm{g} \mathrm{FC} \mathrm{mL}{ }^{-1}$, aproximadamente 3 vezes maior que o controle. 


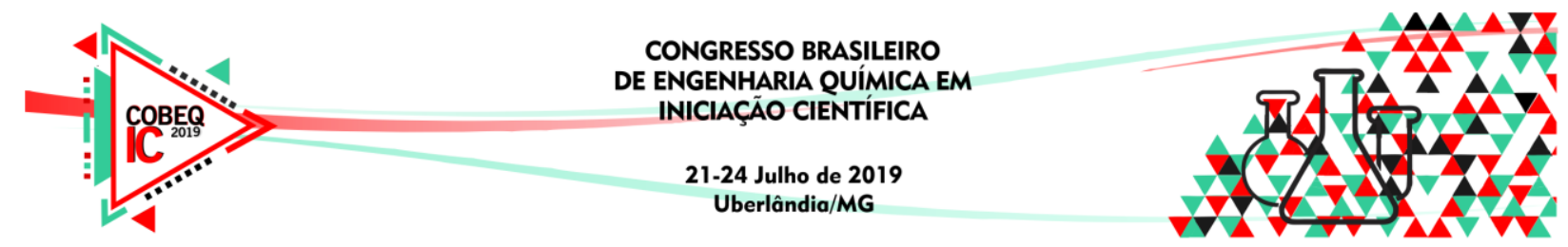

Figura 2 - Crescimento celular obtido dos cultivos de Anabaena variabilis utilizando meios com diferentes fontes de carbono com inóculo de $20 \%$ em 8 dias. Controle $(\bigcirc)$, Glicose $1 \mathrm{~g} / \mathrm{L}(*)$, Lactose $1 \mathrm{~g} / \mathrm{L}(\boldsymbol{\Delta})$, Frutose $1 \mathrm{~g} / \mathrm{L}(\diamond)$, Sacarose $1 \mathrm{~g} / \mathrm{L}(\bullet)$, Galactose $1 \mathrm{~g} / \mathrm{L}$ (匚).

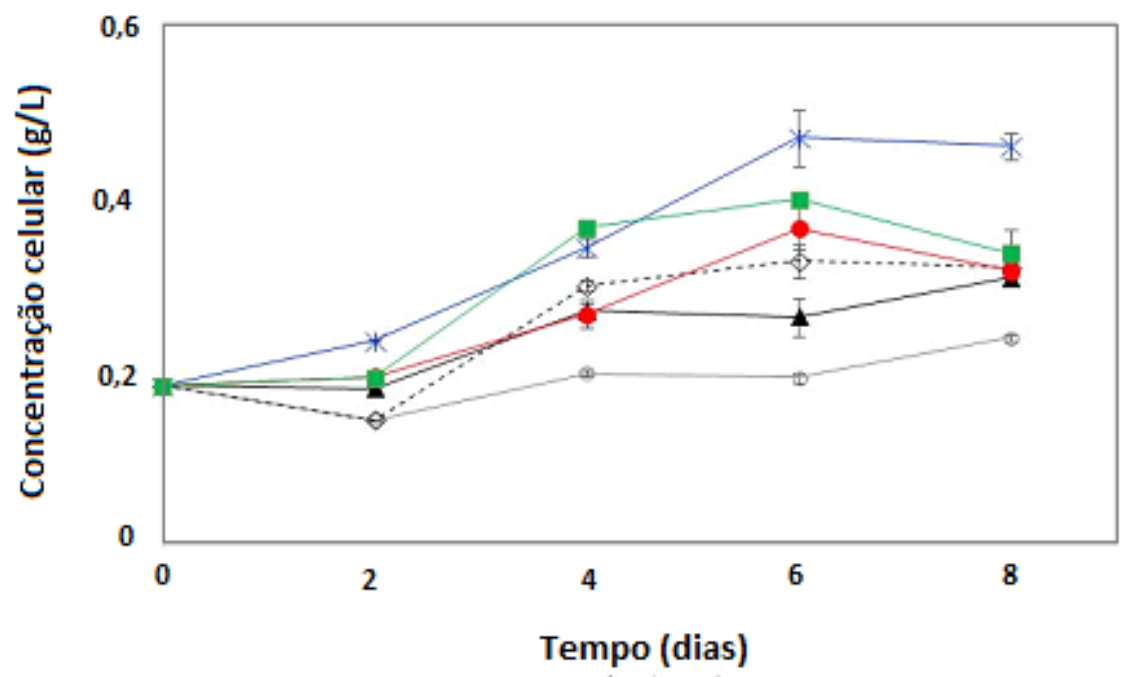

Figura 3 - Concentração de FC dos cultivos de Anabaena variabilis utilizando meios com diferentes fontes de carbono com inóculo de $20 \%$ em 8 dias. Controle ( $\square)$, Glicose $1 \mathrm{~g} / \mathrm{L}$ $(\square)$, Lactose $1 \mathrm{~g} / \mathrm{L}(\square)$, Frutose $1 \mathrm{~g} / \mathrm{L}(\square)$, Sacarose $1 \mathrm{~g} / \mathrm{L}(\square)$, Galactose $1 \mathrm{~g} / \mathrm{L}$ ( $\square$ ). Letras diferentes entre as colunas representam diferença estatística do Teste Tukey com $p<0,05$.

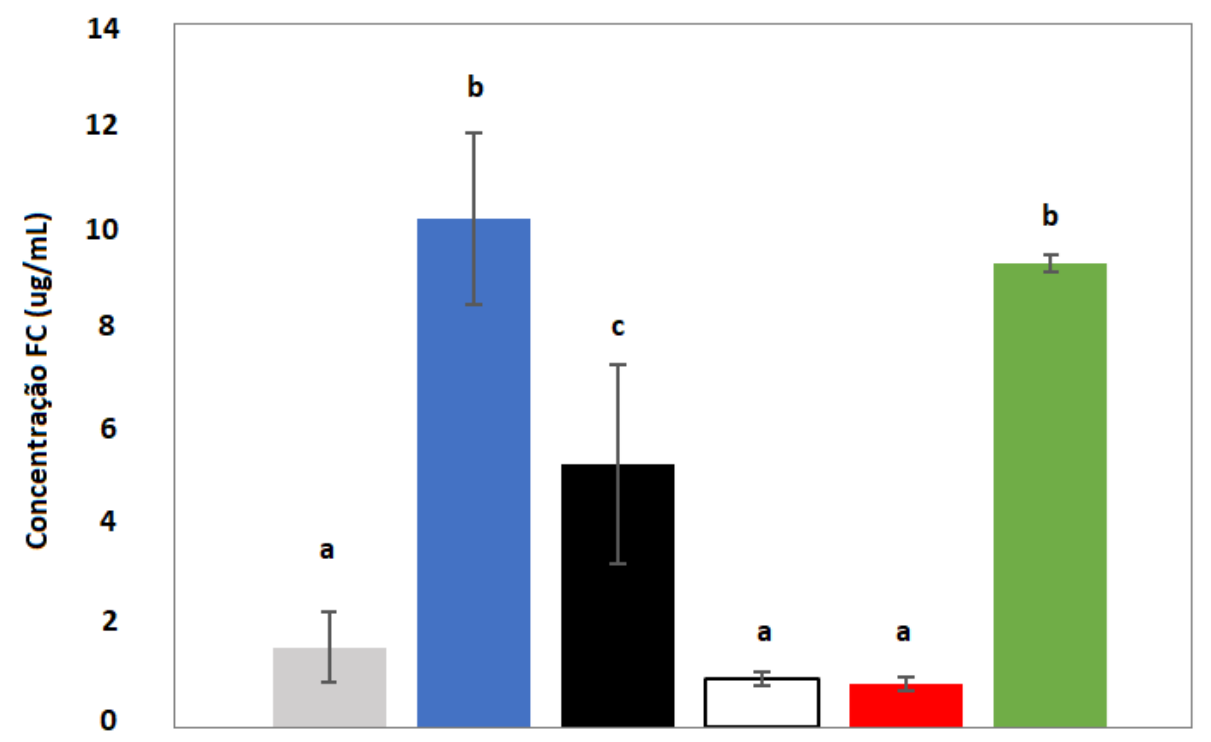

Os meios com adição de frutose e sacarose, não influenciaram significativamente na produção de FC ao final do experimento. Isso mostra que o consumo da fonte de carbono não indica uma maior produção de ficocianina (Figura 3) e produção de biomassa (Figura 2), uma vez que a frutose foi o açúcar mais consumido, indicando que diferentes rotas metabólicas foram utilizadas pela cianobactéria em função da fonte de carbono. 


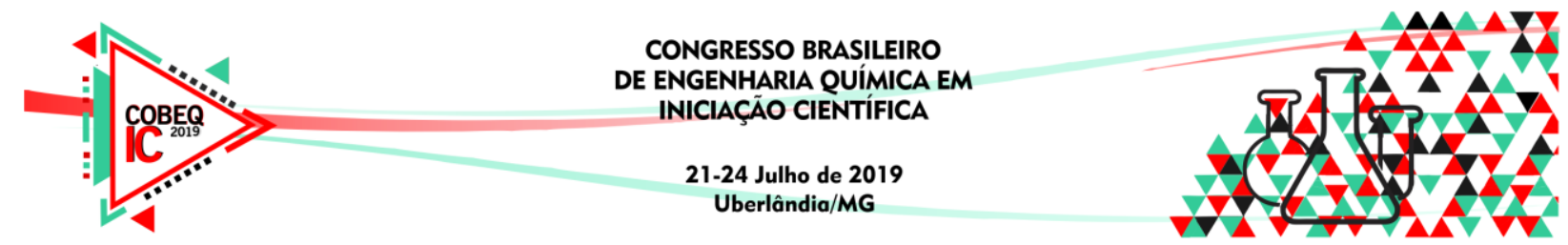

Segundo Khattar et al. (2015), o cultivo de Anabaena fertilissima em meio BG11 0 com suplementação de diferentes tipos de açúcares favoreceu a produção de FBP e apresentou melhores resultados para a produção de FC quando utilizado sacarose a $5 \mathrm{~g} / \mathrm{L}$. Ao comparar este resultado destes autores com o presente trabalho, observa-se que a espécie de cianobactéria também é uma variável a ser considerada na avaliação da produção de FC, sendo que as espécies podem ter metabolismos diferentes de acordo com a composição do meio

Estes dados de produção de FC com apenas uma variação, a fonte de carbono, é um passo para etapas futuras com a combinação e diversos fatores que influenciam a produção de ficocianina, tais como o pH do meio, suplementação de fonte de nitrogênio, açúcar e iluminação de culturas com diferentes cores de luz.

\section{CONCLUSÃO}

O presente trabalho indica que a Anabaena variabilis cultivada em diferentes meios sofre alteração no crescimento celular, na produção de ficobiliproteínas e na capacidade de assimilar açúcares distintos. Conclui-se que a melhor condição é o meio $\mathrm{BG} 11_{0}$ com suplementação de glicose, pois favoreceu a produção de FC (6 vezes) e o aumento da concentração celular ( 2 vezes) durante 8 dias.

\section{AGRADECIMENTOS}

Os autores agradecem ao CNPq, CAPES e FAPEMIG pelo apoio e incentivo à pesquisa.

\section{REFERÊNCIAS}

BORSARI, R. R. J.; MORIOKA, L. R.; RIBEIRO, M. L. L.; BUZATO, J. B. Mixotrophic growth of Nostoc sp. on glucose, sucrose and sugarcane molasses for Phycobiliprotein production. Acta Sci. Biol. Sci., v. 29 (1), p. 9-13, 2007.

COTTAS, A. G. Avaliação do processo de produção de ficobiliproteínas de cianobactérias e purificação por sistemas aquosos bifásicos. Dissertação de Mestrado. Engenharia Química, Universidade Federal de Uberlândia - UFU, 2019.

JACINAVICIUS, F. R.; GAMA JUNIOR, W. A.; AZEVEDO, M. T. P.; SANTANNA, C. Manual para cultivo de cianobactérias. São Paulo: Secretaria do Meio Ambiente, 2013.

KHATTAR, J. I. S., KAUR, S., KAUSHAL, S., SINGH, Y., SINGH, D. P., RANA, S., GULATI, A. Hyperproduction of phycobiliproteins by the cyanobacterium Anabaena fertilissima PUPCCC 410.5 under optimized culture conditions. Algal Res., v. 12, p. 463-469, 2015.

LIU, Y., FENG, Y., LUN, J. Aqueous two-phase countercurrent distribution for the separation of c-phycocyanin and allophycocyanin from Spirulina platensis. Food Bioprod Process, v. 90(2), p. 111-117, 2012. 


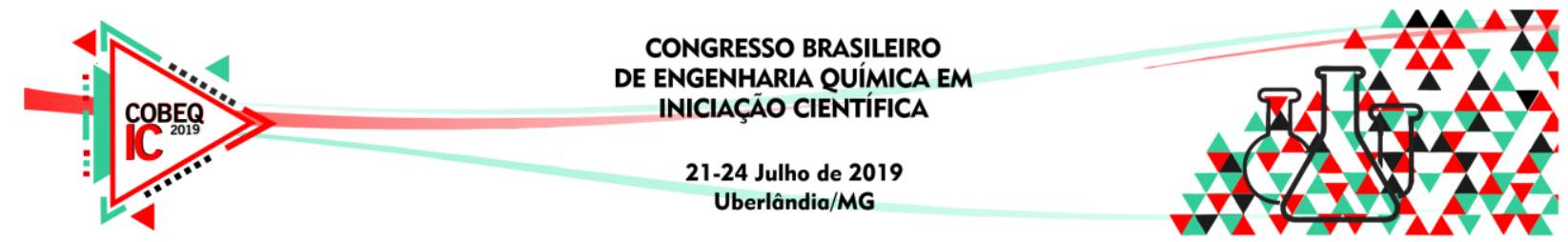

MANIRAFASHA, E., NDIKUBWIMANA, T., ZENG, X., LU, Y., JING, K. Phycobiliprotein: Potential microalgae derived pharmaceutical and biological reagent. Biochem. Eng. J., v. 109, p. 282-296, 2016.

PAGELS, F., GUEDES, A. C., AMARO, H. M., KIJJOA, A., VASCONCELOS, V. Phycobiliproteins from cyanobacteria: Chemistry and biotechnological applications. Biotechnology Advances. v. 37(3), p. 422-443, 2019.

RIZZO, R. F., SANTOS, B. DO N. C. DOS, CASTRO, G. F. P. DA S. DE, PASSOS, T. S., NASCIMENTO, M. DE A., GUERRA, H. D., LIMA-ARAÚJO, K. G. DE. Production of phycobiliproteins by Arthrospira platensis under different lightconditions for application in food products. Food Sci. Technol. (Campinas), v. 35(2), p. 247-252, 2015. 